\title{
The unleashing of the immune system in COVID-19 and sepsis: the calm before the storm?
}

\author{
Salvatore Bellinvia ${ }^{1} \odot$ Christopher J. Edwards ${ }^{1} \cdot$ Matteo Schisano $^{2} \cdot$ Paolo Banfi $^{3} \cdot$ Matteo Fallico $^{4} \cdot$ Paolo Murabito $^{5}$
}

Received: 11 May 2020 / Revised: 19 May 2020 / Accepted: 22 May 2020 / Published online: 28 May 2020

(c) Springer Nature Switzerland AG 2020

\begin{abstract}
The novel coronavirus disease 2019 (COVID-19) pandemic caused by severe acute respiratory syndrome coronavirus 2 (SARS-CoV-2) is sorely testing health care systems and economies around the world and is rightly considered as the major health emergency in a century. Despite the course of the disease appearing to be mild in many cases, a significant proportion of symptomatic patients develop pneumonia requiring hospitalisation or progress to manifest respiratory complications leading to intensive care treatment. Potential interventions for SARS-CoV2-associated pneumonia are being tested, some of which holding promise, but as of today none of these has yet demonstrated outstanding efficacy in treating COVID-19. In this article, we discuss fresh perspectives and insights into the potential role of immune dysregulation in COVID-19 as well as similarities with systemic inflammatory response in sepsis and the rationale for exploring novel treatment options affecting host immune response.
\end{abstract}

Keywords Covid-19 $\cdot$ Immune dysregulation $\cdot$ Inflammatory receptors $\cdot$ Cytokine storm $\cdot$ Sepsis $\cdot$ Checkpoint inhibitors

\section{Introduction}

The world population has experienced the third outbreak of a coronavirus infection with zoonotic origin after Severe Acute Respiratory Syndrome (SARS) and Middle East Respiratory Syndrome (MERS) [1,2]. The causative agent, a positive-sense single-stranded RNA virus, was identified in Wuhan, China, at the end of 2019 and named as

Responsible Editor: Anatoliy Kubyshkin.

Salvatore Bellinvia

salvo.bellinvia@libero.it

1 Department of Rheumatology and NIHR Clinical Research Facility, University Hospital Southampton NHS Foundation Trust, Southampton, UK

2 Department of Clinical and Experimental Medicine, Regional Referral Centre for Rare Lung Diseases, A.O.U. "Policlinico-Vittorio Emanuele", University of Catania, Catania, Italy

3 IRCCS Fondazione Don Carlo Gnocchi, Milan, Italy

4 Ophthalmology Department, A.O.U. "Policlinico-Vittorio Emanuele", University of Catania, Catania, Italy

5 Department of Anaesthesia and Intensive Care, University Hospital "G.Rodolico", University of Catania, via Santa Sofia 78, 95123 Catania, Italy
SARS-CoV-2 [3]. The understanding of the transmission as well as of the clinical, biochemical and radiographic features of disease caused by this viral infection (COVID-19) has progressed almost as rapidly as the spread of the virus [4]. The clinical picture typically includes fever, cough, anosmia, dysgeusia, fatigue, myalgia, gastrointestinal symptoms such as loss of appetite, nausea, diarrhoea and involvement of the lower respiratory tract with dyspnoea in moderateto-severe cases [5]. Biochemical findings of lymphopenia are common and the majority of fatalities appear to be seen within the elderly population and patients with coexisting health conditions, including obesity, with either an aged or dysfunctional immune system [6]. Men with COVID-19 and underlying illness tend to be more at risk for worse disease outcomes than women [7]. Nevertheless, pneumonia requiring hospitalisation and respiratory complications can be not infrequently seen in young adults and healthy individuals. The high fatality rate among patients developing respiratory failure as well as the insufficiency of intensive care facilities and personal protective equipment for healthcare professionals have represented the most important challenges for most national health services in the fight against COVID-19 since the emerging of the outbreak.

General immunopathogenic mechanisms of COVID-19 have been characterized or derived from lessons learnt from 
SARS and MERS epidemics [8, 9] and significant efforts are in progress to develop either effective vaccines or antibodybased drugs which represent the most promising therapies [10]. However, until the former preventive or specific measures are made available, there is a desperate need for effective treatment options able to improve the disease outcome in most severe cases. To date, no therapeutic agents have been licensed to treat COVID-19 and the current management around the world has involved supportive care, and notably in moderate to critical cases, off-label and compassionate use of antivirals, antimalarial or anti-cytokine therapies approved for other indications [11]. Overall an increasing research effort is needed to increase awareness and understanding of immune responses in COVID-19 with particular regard to the mechanisms of virus immune evasion and uncontrolled systemic inflammatory response leading to acute respiratory distress syndrome (ARDS) and multiple organ failure.

\section{The immune-mediated inflammatory hypothesis of COVID-19 and implications for treatment}

The interaction between coronaviruses and the host immune system is central with a view to explaining the onset and the persistence of immune-mediated lung inflammatory injury [12]. Upon virus recognition, the responses associated with coronaviruses are initiated by the innate immune system and continued with the triggering of a prolonged adaptive immune response. It is worth noting that "inflammatory receptors", including Toll-like receptors (TLRs), can recognize pathogen components as inflammatory ligands and are expressed not only in leukocytes but also in vascular endothelial cells, myocardiocytes and alveolar epithelial type 2 cells (AT2), the latter also showing high expression of angiotensin-converting enzyme 2 (ACE2) receptor which has been identified as the functional receptor for SARS-CoV-2 and SARS coronavirus previously [13, 14]. Cells expressing inflammatory receptors are able to trigger intracellular signaling systems to produce mediators such as inflammatory cytokines and chemokines and are also defined as "alert cells". The aberrant activation of alert cells after stimulation of TLRs or other inflammatory receptors might offer a better explanation for the mechanisms underlying the magnitude and amplification of a systemic inflammatory response and induction of vascular endothelial injury as in the case of systemic inflammatory response syndrome (SIRS)-induced vasculitis [15]. Moreover, TLRs are known to play an important role in the innate immune response, particularly in the initial interaction between pathogens and macrophages, and among these TLR7 and TRL8 are specifically implicated in viral-derived single-stranded RNA
(ssRNA) recognition $[16,17]$. The role of TLRs in triggering a hyper-inflammatory response in COVID-19, therefore, merits further attention as well as would do the potential impact of single nucleotide polymorphisms (SNPs) in the genes coding for TLRs on altered susceptibility and the impaired ability of certain individuals to respond properly to SARS-CoV-2 infection [18].

Studies evaluating T-cell response against severe acute respiratory syndrome coronavirus (SARS-CoV) in humans have observed that CD8 $+\mathrm{T}$-cell responses were greater in magnitude than the CD4+T-cell [19]. Previous studies in SARS also focused on the role of the adaptive immune response of T-cells in viral clearance which is just as important as the innate immune response. T-cells undoubtedly have a central pathogenetic role in coronavirus infections and dysregulation of their response is crucial in determining the risk of overwhelming and uncontrolled inflammation resulting from the release of large amounts of pro-inflammatory cytokines which has been referred to as a "cytokine storm' [20]. On the other hand, inefficient immune activation and a poor virus-specific T-cell response underlay severe disease in SARS-CoV in animal models [21]. T cells can, therefore, play a pathological role but also in the first instance a protective function in coronavirus infections.

The pathogenesis of COVID-19-associated pneumonia is not yet fully understood but studies in hospitalised patients, with a focus on severe cases, have revealed high serum levels of pro-inflammatory cytokines including interleukin-(IL)-6 and chemokines secreted by immune effector cells [5]. These findings are similar to those seen in other coronavirus infections where elevated levels of these cytokines are detected in the serum of severe cases compared to those with mild to moderate disease [22]. The common pathological event arising from a cytokine storm is a violent inflammatory response ultimately leading to ARDS and often fatal multiple organ failure. This is confirmed by lung pathological features of COVID-19 which greatly resemble those seen in other coronavirus infections and often show bilateral diffuse alveolar damage [23]. While pathological characteristics unfold at a rapid pace, early observations also seem to draw the attention to features of coagulopathy and thromboembolism with the presence of antiphospholipid antibodies (aPL) in critically ill patients [24], thus meaning vascular endothelial dysfunction with a pathogenetic and clinical picture recalling catastrophic antiphospholipid syndrome (APS) might play a significant role in severe cases [25]. It is clear that these clinical and pathological findings are sometimes difficult to distinguish from other major causes of multiple organ thromboses including disseminated intravascular coagulation (DIC) and thrombotic microangiopathies (TMA). Moreover, many infections may be accompanied by increases in aPL and clinical manifestations of the APS [26]. Regardless, these observations have suggested that anticoagulant treatment, 
mainly with low molecular weight heparin (LMWH), might be associated with a better prognosis in severe COVID-19 patients although its efficacy remains to be validated [27].

Another key element in the inflammatory hypothesis of COVID-19 is the accumulating evidence for a central role of overwhelming macrophage activation and a cytokine profile resembling secondary haemophagocytic lymphohistiocytosis (sHLH) seems to be associated with COVID-19 disease severity [28]. For this reason, treatment of cytokine storm using approved therapies with proven safety profiles has a strong theoretical rationale to address the immediate need to reduce mortality [28]. A multicentre, randomised controlled trial of Tocilizumab, an anti-IL-6 receptor monoclonal antibody licensed for rheumatoid arthritis and chimeric antigen receptor (CAR) T cell-induced cytokine release syndrome (CRS), in patients with COVID-19 associated pneumonia and elevated IL- 6 is currently on-going in China and preliminary data have been encouraging [29]. Tocilizumab is also being used outside mainland China and a clinical trial of 330 patients has been authorized by the Italian Medicines Agency (AIFA) [30]. Baricitinib, a Janus kinase (JAK) inhibitor, seems to affect both inflammation and cellular viral entry in COVID-19 and trials are being developed in a patient population with COVID-19 acute respiratory disease. A similar argument is being considered for use of Ruxolitinib, another selective JAK inhibitor [31].

Overall, since the emerging of the outbreak, a number of clinical trials investigating safety and efficacy of up to sixteen different molecules have been authorised in an attempt to improve disease outcome particularly in hospitalized patients [32]. Notably, on the basis of such an increasing evidence for a central role of the uncontrolled inflammatory response in the disease pathogenesis, many of these studies pertain to anti-cytokine treatment strategies. However, the individual contribution of various cytokines to the pathogenesis of lung damage and systemic complications in COVID-19, including the potential role of secreted proteins involved in extracellular matrix remodelling and fibrogenesis, is not yet entirely understood. For instance, IL-17 levels seem to correlate with increased lung injury scores and greater protein-rich inflammatory lung infiltration and this might be relevant to the case of considering a further treatment option in COVID-19, given the availability of approved biological treatment formulations targeting this cytokine in other indications [33].

\section{Dysregulation of immune response in COVID-19 and sepsis}

Despite the ultimate outcome of an uncontrolled inflammatory response leading to respiratory and systemic complications appears to be well characterized, the pathogenesis of
COVID-19 still holds some darker sides in the stages preceding the onset of the cytokine storm, in an early phase of the infection. The higher mortality rate in the elderly population, in contrast to the reduced number of severe cases among young adults and the rarity of symptomatic cases in children when the innate immune system is highly effective, clearly indicates that pathogenic features are augmented in the setting of a sub-optimally efficient immune response, which is predictable in a more vulnerable and aged patient group [34, 35].

Similarly to immune-mediated respiratory complications in COVID-19, immune dysregulation associated with hyper-inflammation is a common finding in intensive care patients with sepsis [36]. By definition, sepsis is the result of a dysregulated immune response to an infection with harmful host response and impaired immune homeostasis. This is reflected in a clinical phenotype characterized by features of uncontrolled systemic inflammation which finds its biochemical counterpart in a storm of pro-inflammatory cytokines, such as interleukin (IL-6), IL-1 and tumour necrosis factor alpha (TNF- $\alpha)$. On the other side of the coin, there has been increasing interest in the role of immunosuppressive mechanisms in causing or exacerbating sepsis, especially T-cell exhaustion, following the varying time of exposure to antigenic stimulation of the invading pathogen. Lymphocyte exhaustion is a well-characterised phenomenon in the context of sepsis and the latter is also often accompanied by acute respiratory failure secondary to ARDS, similarly to complications of COVID-19 associated pneumonia. ARDS shows similar characteristics in both clinical settings as well as sharing the same risk factors such as older age, obesity and cardiovascular disease. Moreover, the clinical course of sepsis-associated ARDS appears to be substantially equivalent to COVID-associated ARDS showing a protracted recovery of pulmonary gas exchange, a prolonged intensive care unit (ICU) stay and elevated mortality [37, 38].

The paradigm of cytokine storm as a lonely protagonist of the pathogenesis in sepsis was revised by the description of an opposing response trying to counterbalance the destructive effects of the cytokine storm and this was defined as compensatory anti-inflammatory response syndrome (CARS) [39]. The goal of this opposing response would be to compensate the overwhelming inflammatory storm and restore homeostasis by means of the release of certain anti-inflammatory cytokines including IL-10. This concept seems to be now widely accepted and its immunological mechanisms have been further characterised over the years but interestingly no clear evidence to support a two-phase model in sepsis pathophysiology was actually found [40]. However, despite some disagreement on simultaneousness of pro- and anti-inflammatory responses, CARS has been credited with an essential role in counter-balancing the detrimental effects of acute hyper-inflammatory over-response. 
The elderly population is at a disproportionately increased risk of sepsis and the same has been observed in COVID19 systemic complications [41]. This is likely due to dysfunction in both arms of immune defence but particularly in adaptive immunity and namely in T-cells capacity to exert an effective response. This phenomenon is also known as "immunosenescense" and is likely to explain the increased risk and higher mortality within the elderly population [42, 43].

\section{Immune therapy in sepsis}

The interest in immune therapies potential in sepsis has been reinvigorated following the advances in cancer immune therapy and awareness that prolonged immune-suppression status in sepsis can leave patients vulnerable to secondary infections and death [44].

Previously, the assumption that the cytokine storm was a primary cause of death in sepsis had been important in an attempt to target specific pro-inflammatory mediators (TNF- $\alpha$ and IL-1 $\beta$ ) with doubtful efficacy, possibly due to non-patient specific approach [45]. Conversely, IL-6 blockade strategies seem to hold more promise with encouraging results on acute lung injury and acute kidney injury in rat models of sepsis and may constitute a novel therapeutic strategy for various types of conditions where cytokine storm is involved [46-48]. Recent findings also suggest the potential benefit of anakinra and tocilizumab in patients with sHLH/macrophage activation syndrome (MAS) associated with rheumatic and non-rheumatic conditions [49, 50].

The overwhelming inflammatory response in sepsis is, however, accompanied by an anti-inflammatory process which correlates with poor outcomes [51]. It is, therefore, clear that proper balance between the often competing proand anti-inflammatory pathways determine the fate of the individual as well as the severity of sepsis-induced immunosuppression. As anticipated a biphasic trend with an initial pro-inflammatory phase followed by an anti-inflammatory phase has been generally dismissed in recent reports whereas it has been suggested that the two phases are likely to coexist $[52,53]$.

A range of biomarkers of sepsis-induced immune suppression has been identified given that the utility of cytokines is limited to this aim [43]. To date, monocyte HLA-DR expression has revealed to be the best biomarker for the overall immune function in sepsis and has been largely used in clinical trials, particularly after the development of systems able to minimise inter-laboratory variability and the introduction of rapid, automated devices able to provide a point of care testing [54]. Furthermore, pre-clinical and clinical studies have shown that inhibitory immune checkpoint molecules, including programmed death-1 (PD-1), programmed death ligand-1 (PD-L1), cytotoxic T lymphocyte antigen-4 (CTLA4), T cell membrane protein-3 (TIM-3) and lymphocyte activation-gene-3 (LAG-3) are upregulated during the course of sepsis and their expression or engagement on various immune cells, and especially on $\mathrm{T}$ cell surface, has shown to play a relevant role in reducing pathogen clearance and immune system competence, contributing overall to exhaustion-induced immunosuppression [55]. On the grounds of these findings, immune cell checkpoints have become a potential target during sepsis in an attempt to restore host immune response with the PD-1/PD-L1 axis being by far the most studied immune checkpoint interaction [56]. Checkpoint inhibitors (CPIs) can sustain lymphocyte activation and benefit host defence in select clinical contexts. Monoclonal antibodies (mAb) blocking PD-1, its ligand PD-L1 or CTLA-4 augment tumour-reactive cytotoxic T-cell function and are currently FDA-approved treatments for several cancers [57]. Interestingly, PD-L1 blockade seemed to improve survival in experimental sepsis by attenuating T-cell apoptosis as well as enhancing pathogen clearance, and of considerable importance, decreasing systemic inflammation [58]. A recent meta-analysis examining the benefit of immune therapy in preclinical studies showed CPIs improved host defence and survival during bacterial sepsis providing one basis for phase 1 CPI sepsis trials [59]. Moreover, these medications have proven to be safe and active in real-life practice even in challenging populations who would have been ineligible for clinical trials, including patients with dysregulated or compromised immune function, significant co-morbidities or in the elderly population [60]. Careful consideration, however, will be certainly needed in introducing these treatment strategies because of the hypothetical risk of a detrimental effect of unleashing the immune system functions in the setting of a systemic inflammatory response [61].

\section{Discussion}

The pathogenesis of COVID-19 is still poorly understood although it appears to share similar mechanisms to SARS-CoV and MERS-CoV infections. However, its pandemic dimension has posed an unprecedented challenge to health systems and this has generated tireless efforts within the research community to get a better understanding of its molecular immune features [8]. The wide geographical spread of the infection has also unveiled populations at higher risks to develop serious respiratory complications associated with poor outcomes and these often overlap with subjects with a dysfunctional immune system [62]. Most of the research to date has focused on the adaptive as compared to humoral response and this leaves a lot of unanswered questions on the features of 
the early phase of infection. For instance, very little is known about mechanisms of immune evasion and weaknesses in host immune response which prevent effective viral clearance as well as factors able to trigger an overwhelming inflammatory comeback and cytokine storm in a subset of patients. With this in mind, the role of TLRs signaling in regulating SARS-CoV-2 pathogenesis should be further investigated as this might offer better insight into the early stages involved in the immune-mediated hyper-inflammatory response. Nevertheless, the consideration of the cytokine storm as the lone protagonist of immune-mediated complications in COVID-19 might be reductive and unable to fully explain how pathogenic features are more likely to be enhanced in subjects with a sub-optimally efficient immune response. Similarly to sepsis, immune dysregulation associated with both hyperinflammation and immunosuppression might be a common finding in intensive care patients with COVID-19.

Recent advances in immunotherapy in cancer have raised the possibility that host-directed therapy (HDT) via immune checkpoint blockade might have therapeutic implications for infectious diseases including sepsis $[56,63]$. In patients with chronic viral infections CD8 T-cell exhaustion, mediated by PD-1 expression on the cell surface, is an important cause of impairment in antiviral effector functions and clearance of infected host cells, thus prompting clinical evaluation of strategies to enhance immune response $[64,65]$. Therapies based on immune checkpoints inhibition have not been tested in infections as they have in cancer [66] and very little is known about their potential in acute viral infections such as coronavirus infections. However, it is clear that successful pathogens evolve mechanisms to evade and constrain protective host responses and this might be particularly true for COVID-19 in a subset of patients who have a dysregulated immune function, though not to the extent to prevent them from sparking off a cytokine storm leading to immune-mediated inflammatory lung injury. A better understanding and further characterization of cell surface expression of checkpoint inhibitors and other immunomodulatory markers on immune cells during the course of COVID-19 is advisable to understand how dysregulation of immune cells may inform the clinical risk in these patients. Increased knowledge about molecular immune pathogenesis in COVID-19 is also desirable for possible consideration of HDT with a view to restoring effector T-cell responses, particularly in the early phase of infection. However, the greatest potential is likely to lie in a combination, multi-targeted therapy at different stages of severity, ideally guided by point of care testing, so as to allow a more accurate and patient-specific approach to immunomodulation in COVID-19 patients.

\section{Conclusions}

The novel COVID-19 pandemic has posed unparalleled challenges to health systems worldwide and called upon the scientific community in an unprecedented research effort and race to develop specific antiviral therapies and effective vaccines. Containment and delay strategies are essential at this stage to take the hit, keep closed ranks and gain time. Nevertheless, until specific treatments are made available, there is a desperate need for therapeutic options able to improve the disease outcome in most severe cases.

Current management of COVID-19 is a largely supportive and respiratory failure from ARDS associated with multi-organ failure is the main cause of mortality in a subset of patients. There is increasing evidence that individuals with poor outcomes have a cytokine storm, in many ways similar to sepsis, hence targeting hyper-inflammation has understandably taken the centre stage at this moment in time. However, very little is known about the molecular pathogenetic mechanisms involving the innate immune response in the stages preceding the onset of the cytokine storm. Further research is also needed to understand the underlying molecular pathways potentially hindering a balanced and effective immune response against SARS$\mathrm{CoV}-2$ in more severe cases and the potential for exploring novel immunoadjuvant treatment strategies.

\section{References}

1. de Wit E, van Doremalen N, Falzarano D, et al. SARS and MERS: recent insights into emerging coronaviruses. Nat Rev Microbiol. 2016;14:523-34.

2. Zhou P, Yang XL, Wang XG, et al. A pneumonia outbreak associated with a new coronavirus of probable bat origin. Nature. 2020;579:270-3. https://doi.org/10.1038/s41586-020-2012-7.

3. World Health Organization. Coronavirus disease (COVID-19) outbreak. https://www.who.int/emergencies/diseases/novel -coronavirus-2019. Accessed Feb 2020

4. Li Q, Guan X, Wu P, et al. Early Transmission Dynamics in Wuhan, China, of Novel Coronavirus-Infected Pneumonia. N Engl J Med. 2020;382(13):1199-207. https://doi.org/10.1056/ NEJMoa2001316.

5. Huang C, Wang Y, Li X, et al. Clinical features of patients infected with 2019 novel coronavirus in Wuhan, China. Lancet. 2020;395(10223):497-506.

6. Chen N, Zhou M, Dong X, et al. Epidemiological and clinical characteristics of 99 cases of 2019 novel coronavirus pneumonia in Wuhan, China: a descriptive study. Lancet. 2020;395(10223):507-13.

7. Jin JM, Bai P, He W, et al. Gender differences in patients with COVID-19: focus on severity and mortality. Front Public Health. 2020;29(8):152.

8. Li X, Geng M, Peng Y, et al. Molecular immune pathogenesis and diagnosis of COVID-19. J Pharm Anal. 2020;10(2):102-8. https://doi.org/10.1016/j.jpha.2020.03.001. 
9. Prompetchara E, Ketloy C, Palaga T. Immune responses in COVID-19 and potential vaccines: lessons learned from SARS and MERS epidemic. Asian Pac J Allergy Immunol. 2020;38(1):1-9. https://doi.org/10.12932/AP-200220-0772.

10. Shanmugaraj B, Siriwattananon K, Wangkanont K, et al. Perspectives on monoclonal antibody therapy as potential therapeutic intervention for Coronavirus disease-19 (COVID-19). Asian Pac J Allergy Immunol. 2020;38(1):10-8. https://doi.org/10.12932/ AP-200220-0773

11. Zhang L, Liu Y. Potential interventions for novel coronavirus in China: A systematic review. J Med Virol. 2020;92(5):479-90.

12. Li G, Fan Y, Lai Y, et al. Coronavirus infections and immune responses. J Med Virol. 2020;92(4):424-32.

13. Zhang H, Penninger JM, Li Y, et al. Angiotensin-converting enzyme 2 (ACE2) as a SARS-CoV-2 receptor: molecular mechanisms and potential therapeutic target. Intensive Care Med. 2020;46(4):586-90.

14. Hamming I, Timens W, Bulthuis ML, et al. Tissue distribution of ACE2 protein, the functional receptor for SARS coronavirus. A first step in understanding SARS pathogenesis. J Pathol. 2004;203(2):631-7.

15. Matsuda N. Alert cell strategy in SIRS-induced vasculitis: sepsis and endothelial cells. J Intensive Care. 2016;23(4):21.

16. Li Q, Cherayil BJ. Role of toll-like receptor 4 in macrophage activation and tolerance during salmonella enterica serovar typhimurium infection. Infect Immun. 2003;71(9):4873-82.

17. Takeda K, Akira S. Toll-like receptors in innate immunity. Int Immunol. 2005;17(1):1-14.

18. Schröder NW, Schumann RR. Single nucleotide polymorphisms of toll-like receptors and susceptibility to infectious disease. Lancet Infect Dis. 2005;5(3):156-64.

19. Li CK, Wu H, Yan H, et al. T cell responses to whole SARS coronavirus in humans. J Immunol. 2008;181(8):5490-500.

20. Janice Oh HL, Ken-En Gan S, Bertoletti A, et al. Understanding the $\mathrm{T}$ cell immune response in SARS coronavirus infection. Emerg Microbes Infect. 2012;1(9):e23.

21. Zhao J, Zhao J, Van Rooijen N, et al. Evasion by stealth: inefficient immune activation underlies poor $\mathrm{T}$ cell response and severe disease in SARS-CoV-infected mice. PLoS Pathog. 2009;5(10):e1000636.

22. Min CK, Cheon S, Ha NY, et al. Comparative and kinetic analysis of viral shedding and immunological responses in MERS patients representing a broad spectrum of disease severity. Sci Rep. 2016;6:25359.

23. Xu Z, Shi L, Wang Y, et al. Pathological findings of COVID19 associated with acute respiratory distress syndrome. Lancet Respir Med. 2020;8(4):420-2. https://doi.org/10.1016/S2213 -2600(20)30076-X.

24. Zhang Y, Xiao M, Zhang S, Zhang S, Li Y. Coagulopathy and antiphospholipid antibodies in patients with Covid-19. N Engl J Med. 2020;382(17):e38. https://doi.org/10.1056/NEJMc2007575.

25. Espinosa G, Cervera R, Font J, Asherson RA. The lung in the antiphospholipid syndrome. Ann Rheum Dis. 2002;61(3):195-8.

26. Shoenfeld Y, Blank M, Cervera R, Font J, Raschi E, Meroni PL. Infectious origin of the antiphospholipid syndrome. Ann Rheum Dis. 2006;65(1):2-6.

27. Tang N, Bai H, Chen X, et al. Anticoagulant treatment is associated with decreased mortality in severe coronavirus disease 2019 patients with coagulopathy. J Thromb Haemost. 2020. https://doi. org/10.1111/jth.14817.

28. Mehta P, McAuley F, Brown M, et al. COVID-19: consider cytokine storm syndromes and immunosuppression. Lancet. 2020. https://doi.org/10.1016/S0140-6736(20)30628-0.

29. Chinese Clinical Trial Registry. A multicenter, randomized controlled trial for the efficacy and safety of tocilizumab in the treatment of new coronavirus pneumonia (COVID-19). Feb 13,
2020. https://www.chictr.org.cn/showprojen.aspx?proj=49409. Accessed 6 Mar 2020.

30. AIFA e Istituto Nazionale per lo studio e la cura dei tumori di Napoli avviano uno studio per l'utilizzo di Tocilizumab nella malattia COVID 19. https://www.aifa.gov.it/web/guest/-/aifa-eistituto-nazionale-per-lo-studio-e-la-cura-dei-tumeri-di-napol i-avviano-uno-studio-per-1-utilizzo-di-tocilizumab-nella-malat tia-covid-19. Accessed Mar 2020

31. Richardson P, Griffin I, Tucker C, et al. Baricitinib as potential treatment for 2019-nCoV acute respiratory disease. Lancet. 2020;395:e30-31.

32. Clinical Trials-COVID-19 (AIFA) https://www.aifa.gov.it/speri mentazioni-cliniche-covid-19. Accessed May 2020

33. Pacha O, Sallman MA, Evans SE. COVID-19: a case for inhibiting IL-17? Nat Rev Immunol. 2020. https://doi.org/10.1038/s4157 7-020-0328-Z.

34. de Heredia FP, Gómez-Martínez S, Marcos A. Obesity, inflammation and the immune system. Proc Nutr Soc. 2012;71(2):332-8.

35. Fuentes E, Fuentes M, Alarcón M, et al. Immune system dysfunction in the elderly. An Acad Bras Cienc. 2017;89(1):285-99.

36. Gillis A, Beil M, Halevi-Tobias K, et al. Alleviation of exhaustioninduced immunosuppression and sepsis by immune checkpoint blockers sequentially administered with antibiotics-analysis of a new mathematical model. Intensive Care Med Exp. 2019;7(1):32.

37. Rubenfeld GD, Caldwell E, Peabody E, et al. Incidence and outcomes of acute lung injury. N Engl J Med. 2005;353(16):1685-93.

38. Wu C, Chen X, Cai Y, Xia J, et al. Risk factors associated with acute respiratory distress syndrome and death in patients with coronavirus disease 2019 pneumonia in Wuhan, China. JAMA Intern Med. 2020. https://doi.org/10.1001/jamainternmed.2020.0994.

39. Bone RC. Sir Isaac Newton, sepsis, SIRS, and CARS. Crit Care Med. 1996;24(7):1125-8.

40. Gomez HG, Gonzalez SM, Londoño JM, et al. Immunological characterization of compensatory anti-inflammatory response syndrome in patients with severe sepsis: a longitudinal study. Crit Care Med. 2014;42(4):771-80.

41. Martin GS, Mannino DM, Moss M. The effect of age on the development and outcome of adult sepsis. Crit Care Med. 2006;34(1):15-211.

42. Pawelec G. Age and immunity: What is "immunosenescence"? Exp Gerontol. 2018;105:4-9.

43. Martín S, Pérez A, Aldecoa C. Sepsis and immunosenescence in the elderly patient: a review. Front Med (Lausanne). 2017;28(4):20.

44. Davies R, O'Dea K, Gordon A. Immune therapy in sepsis: Are we ready to try again? J Intensive Care Soc. 2018;19(4):326-44.

45. Fisher CJ Jr, Agosti JM, Opal SM, et al. Treatment of septic shock with the tumor necrosis factor receptor: Fc fusion protein. The Soluble TNF Receptor Sepsis Study Group. N Engl J Med. 1996;334(26):1697-702.

46. Krüttgen A, Rose-John S. Interleukin-6 in sepsis and capillary leakage syndrome. J Interferon Cytokine Res. 2012;32(2):60-5.

47. Ibrahim YF, Moussa RA, Bayoumi AMA. Tocilizumab attenuates acute lung and kidney injuries and improves survival in a rat model of sepsis via down-regulation of NF- $\mathrm{KB} / \mathrm{JNK}$ : a possible role of P-glycoprotein. Inflammopharmacology. 2020;28(1):215-30.

48. Tanaka T, Narazaki M, Kishimoto T. Immunotherapeutic implications of IL-6 blockade for cytokine storm. Immunotherapy. 2016;8(8):959-70.

49. Eloseily EM, Weiser P, Crayne CB, et al. Benefit of anakinra in treating pediatric secondary hemophagocytic lymphohistiocytosis. Arthritis Rheumatol. 2020;72(2):326-34.

50. Zhou S, Qiao J, Bai J, et al. Biological therapy of traditional therapy-resistant adult-onset Still's disease: an evidence-based review. Ther Clin Risk Manag. 2018;14:167-71. 
51. Wu HP, Chen CK, Chung K, et al. Serial cytokine levels in patients with severe sepsis. Inflamm Res. 2009;58(7):385-93.

52. Boomer JS, Green JM, Hotchkiss RS. The changing immune system in sepsis: is individualized immuno-modulatory therapy the answer? Virulence. 2014;5(1):45-56.

53. Gentile LF, Cuenca AG, Efron PA, et al. Persistent inflammation and immunosuppression: a common syndrome and new horizon for surgical intensive care. J Trauma Acute Care Surg. 2012;72(6):1491-501.

54. Demaret J, Walencik A, Jacob MC, et al. Inter-laboratory assessment of flow cytometric monocyte HLA-DR expression in clinical samples. Cytometry B Clin Cytom. Jan-Feb 2013;84(1):59-62. https://doi.org/10.1002/cyto.b.21043 [Epub 14 Sept 2012]

55. Guignant C, Lepape A, Huang X, et al. Programmed death-1 levels correlate with increased mortality, nosocomial infection and immune dysfunctions in septic shock patients. Crit Care. 2011;15(2):R99. https://doi.org/10.1186/cc10112.

56. Patil NK, Guo Y, Luan L, et al. Targeting immune cell checkpoints during sepsis. Int J Mol Sci. 2017;18(11):E2413.

57. Chen L, Han X. Anti-PD-1/PD-L1 therapy of human cancer: past, present, and future. J Clin Invest. 2015;125(9):3384-91.

58. Zhang Y, Zhou Y, Lou J, et al. PD-L1 blockade improves survival in experimental sepsis by inhibiting lymphocyte apoptosis and reversing monocyte dysfunction. Crit Care. 2010;14(6):R220.

59. Busch LM, Sun J, Cui X, et al. Checkpoint inhibitor therapy in preclinical sepsis models: a systematic review and meta-analysis. Intensive Care Med Exp. 2020;8(1):7.
60. Johnson DB, Sullivan RJ, Menzies AM. Immune checkpoint inhibitors in challenging populations. Cancer. 2017;123(11):1904-11.

61. Cavaillon JM, Eisen D, Annane D. Is boosting the immune system in sepsis appropriate? Crit Care. 2014;18(2):216.

62. Guan WJ, Ni ZY, Hu Y, et al. Clinical Characteristics of Coronavirus Disease 2019 in China. N Engl J Med. 2020;382:1708-20. https://doi.org/10.1056/NEJMoa2002032.

63. Rao M, Valentini D, Dodoo E, et al. Anti-PD-1/PD-L1 therapy for infectious diseases: learning from the cancer paradigm. Int $\mathbf{J}$ Infect Dis. 2017;56:221-8.

64. Day CL, Kaufmann DE, Kiepiela P, et al. PD-1 expression on HIV-specific T cells is associated with T-cell exhaustion and disease progression. Nature. 2006;443(7109):350-4.

65. Ye B, Liu X, Li X, et al. T-cell exhaustion in chronic hepatitis B infection: current knowledge and clinical significance. Cell Death Dis. 2015;19(6):e1694.

66. Dyck L, Mills KHG. Immune checkpoints and their inhibition in cancer and infectious diseases. Eur J Immunol. 2017;47(5):765-79.

Publisher's Note Springer Nature remains neutral with regard to jurisdictional claims in published maps and institutional affiliations. 